\title{
La courbe parétienne des revenus et la sociologie économique
}

\section{Philippe Steiner}

\section{(2) OpenEdition}

\section{Journals}

Édition électronique

URL : http://journals.openedition.org/ress/2611

DOI : 10.4000/ress.2611

ISSN : 1663-4446

Éditeur

Librairie Droz

\section{Édition imprimée}

Date de publication : 15 décembre 2013

Pagination : 193-211

ISBN : 978-2-600-01805-0

ISSN : 0048-8046

\section{Référence électronique}

Philippe Steiner, «La courbe parétienne des revenus et la sociologie économique », Revue européenne des sciences sociales [En ligne], 51-2 | 2013, mis en ligne le 01 janvier 2017, consulté le 19 mars 2020. URL : http://journals.openedition.org/ress/2611 ; DOI : https://doi.org/10.4000/ress.2611 


\title{
LA COURBE PARÉTIENNE DES REVENUS ET LA SOCIOLOGIE ÉCONOMIQUE
}

PHILIPPE STEINER

Université de Paris-IV Sorbonne/Institut universitaire de France philippe.steiner@paris-sorbonne.fr

Résumé. Pareto a dégagé une loi importante avec sa courbe de distribution des revenus, dont il donne une interprétation essentiellement politique, notamment contre les socialistes soucieux de plus d'égalité. Dans ce texte, nous montrons qu'elle peut également être considérée comme une pièce importante de la sociologie économique, tant elle permet d'étudier ce qu'il se passe à la «pointe» de la distribution, ainsi que les travaux de Thomas Piketty le montrent.

Mots-clés: Vilfredo Pareto, Thomas Piketty, élites, revenus, sociologie économique, distribution des revenus.

\begin{abstract}
Pareto discovered an important result with his statistical research on the distribution of incomes. His own interpretation of this distribution was cast in political terms in order to fight the socialists willing to get a more egalitarian distribution. This article highlights the fact that Pareto's distribution of incomes is an important piece of his economic sociology that is still very useful for examining what is going on in the upper strata of the incomes elite, as exemplified by Thomas Piketty's works.
\end{abstract}

Keywords: Vilfredo Pareto, Thomas Piketty, elites, economic sociology, incomes distribution. 
Par son ampleur et par sa structure, l'œuvre de Vilfredo Pareto entre de plain-pied dans le domaine que l'on appelle désormais la sociologie économique - terme que lui-même n'a employé qu'à de rares exceptions. Néanmoins, la «courbe des revenus » qu'il met au jour par ses recherches statistiques exemplaires n’a pas été retenue comme une contribution significative de ce domaine. Ce numéro exceptionnel organisé par la revue qui, initialement, a paru sous le nom du grand économiste et sociologue de l'université de Lausanne, offre l'occasion de revenir sur cette courbe et de montrer combien celle-ci est un apport significatif à la sociologie économique.

La première partie rappelle la manière dont Pareto présente la courbe des revenus, les principes et les résultats sur lesquels il met l'accent. La deuxième partie explique la raison pour laquelle la courbe des revenus entre dans le cadre de la sociologie économique telle que Pareto la pratique. La troisième partie examine la pointe de la courbe à la lumière des travaux qui se sont intéressés aux très hauts revenus depuis la fin des années I990; la dernière esquisse une brève comparaison entre le thème parétien de la circulation des élites et le thème wébérien de la sélection.

\section{LA PLACE DE LA COURBE DES REVENUS DANS L'GUVRE DE PARETO}

Dans le Cours d'Économie politique (1964 [1896-1897]), Pareto donne une place tout à fait particulière à la courbe des revenus. La présentation de son remarquable travail de statistiques économiques n’arrive qu’en fin du deuxième volume, précédant le tout dernier chapitre consacré à la physiologie sociale, laquelle se caractérise par la prise en compte de l'ensemble du système social. La courbe des revenus fait donc partie de l'économie appliquée et jouxte ce que Pareto dénommera plus tard la sociologie générale. Cette position appelle deux remarques. D’une part, la lecture du Cours montre que Pareto ne développe pas une théorie pure de la répartition du revenu. En cette matière, la théorie pure se limite à l'exposé des équations d'offre et de demande des services personnels et des capitaux dans le cadre de l'équilibre généralwalrasien; rien n’est dit à propos des conséquences de cet équilibre en termes de répartition des revenus par classes 
sociales ou par types de revenu. Pareto s'en tient à l'idée selon laquelle les différents services producteurs sont rémunérés selon les règles générales de la loi de l'offre et de la demande. Ce faisant, il ne développe pas une théorie pure de la répartition avant d'expliquer ce que seraient les modifications apportées par la prise en compte des passions de l'acteur économique dans le cadre de l'économie politique appliquée. C’est dans un autre sens que Pareto envisage l'impact de la répartition sur l'économie pure : «La répartition des revenus est une des circonstances principales dont il faut tenir compte pour connaître les lois qui régissent réellement l'offre et la demande» (Pareto, I964 [1896-1897], p. 331, §973). Idée qu'il poursuit en esquissant l'effet d'une hausse des impôts - une de ses «bêtes noires» - sur la consommation des biens, et tout particulièrement des biens alimentaires de base, avec l'examen de la loi (empirique) de Gregory King. D’autre part, en plaçant la courbe des revenus à frontière de l'économie appliquée et de la sociologie, Pareto est conduit à mettre l'accent sur la question des inégalités et à lancer une polémique contre les socialistes, Ferdinand Lassalle tout particulièrement. Contre ce dernier, Pareto entend montrer que la répartition des revenus dépend de la nature des hommes et non de l'organisation sociale :

Si les variations de la répartition des richesses suivent les variations de l'organisation économique, c'est à cette organisation que nous devons attribuer une part prépondérante. Enfin, si la répartition de la richesse varie peu pour des contrées, des époques, des organisations différentes, il nous faudra conclure que, sans vouloir négliger les autres causes, nous devons chercher dans la nature de l'homme la cause principale qui détermine le phénomène (ibid., p. 304, §957).

Les données qu'il recueille sur différents pays ou provinces (Angleterre, Prusse, Saxe, Bâle, canton de Vaud, Florence, Pérou) et différentes époques (deuxième moitié du XIX ${ }^{e}$ siècle, milieu du Xv siècle) lui permettent d’aboutir à l'obtention de droites de pentes assez proches pour l'équation estimée $(\log N=\log A-\alpha \log x$; avec $N$ le nombre de personnes ayant un revenu supérieur à $x$ ), ce qui lui permet de conclure que, contrairement à l'affirmation de Lassalle, l'organisation sociale n'intervient pas d'une manière significative dans la répartition des revenus. Il en est de même du hasard, puisque la courbe des revenus diffère de la «courbe des erreurs » (la loi normale). Pareto 
termine son exposé par des considérations un peu brouillonnes sur la tendance à une diminution des inégalités de revenu suite à l'augmentation de la richesse produite et par l'affirmation qu'une telle tendance risque de se briser suite à la «destruction qui en [les richesses] est faite par la protection douanière, les vols des politiciens et le socialisme d’État» (ibid., p. 325, §965).

Dix ans plus tard', dans le Manuel d'Économie politique (1966 [1909]) (la courbe des revenus prend place dans un chapitre consacré à la population. Ce chapitre vise pour l'essentiel à présenter une série de réflexions sur l'inégalité et la hiérarchie. Il est bien sûr question de la mesure de l'inégalité, de la population considérée comme «capital personnel» - ce qui fait le lien avec le chapitre suivant sur les capitaux fonciers et mobiliers - et de la relative stabilité de la courbe des revenus, que Pareto prend, une nouvelle fois, grand soin de distinguer de la loi normale, en soulignant le fait que cette courbe est asymétrique par rapport à la moyenne ou la valeur modale. L'essentiel de son propos tourne cependant autour de quatre thèmes :

Nous venons de mentionner quatre espèces de faits, c'est-à-dire: la hiérarchie - la succession des aristocraties - la sélection - la proportion moyenne de richesse ou de capitaux par habitant. Ces faits sont de beaucoup les plus importants pour déterminer le caractère de la société, c'est-à-dire des autres faits sociaux. Mais, en réalité, ce n'est pas d'un rapport de cause à effet qu'il s'agit. Les premiers faits agissent sur les seconds, mais ceux-ci, à leur tour, réagissent sur ceux-là; et, en définitive, nous sommes en présence d'un rapport de mutuelle dépendance (Pareto, I966 [1909], p. 425, §ı I02).

La référence à la mutuelle dépendance des phénomènes sociaux entraîne l'idée selon laquelle les caractéristiques de la population ont une influence sur les autres faits sociaux qui rétroagissent sur celles-ci. Ce type de considération est un point crucial de sa sociologie économique, comme on l'explicitera plus bas. Néanmoins, Pareto fait à nouveau une lecture politique et polémique

I Dans Les Systèmes socialistes (1965 [1901-1902]), Pareto mobilise à l'occasion sa courbe des revenus, mais sans offrir de développements originaux, sauf, peut-être, lorsqu'il suppose une expropriation des revenus au-delà d'un certain niveau. Cette mesure, dit-il, changerait la distribution des revenus pour quelques générations avant que la forme initiale ne reprenne place, au prix d'une diminution du capital accumulé et, par conséquent, de la richesse produite puisque ce sont les classes riches qui épargnent et accumulent (ibid., p. 158-160). 
(contre les «humanitaires » et les socialistes) de son argument en rapprochant la question de la hiérarchie et de la circulation des élites de celle concernant la propriété et l'action (spoliatrice) du gouvernement :

Si chez nos populations occidentales l'élément de stabilité était exclusivement le résultat de l'institution de la propriété privée et de l'hérédité, il y aurait là une démonstration très forte de la nécessité de diminuer, ou même de supprimer, l'institution de la propriété privée. Il est étrange que les socialistes n’aient pas aperçu l'appui que cette façon de considérer les phénomènes pouvait apporter à leurs théories. Mais l'élément de stabilité qui s'oppose au changement par la sélection est loin d'être exclusivement la conséquence, dans nos sociétés, de l’institution de la propriété privée (Pareto, ibid., p. 43I, §ıI5).

Dans le Traité de sociologie générale (1968 [1916]) la courbe des revenus s'efface plus encore devant l'importance accordée à la circulation des élites et à la dimension politique de la sociologie générale que Pareto entreprend de présenter plus systématiquement. Il rapporte désormais les élites et leur circulation aux résidus, dont les deux premières classes - «l'instinct des combinaisons» et la «persistance des agrégats » - retiennent son attention. S’il est question de répartition dans cet ouvrage, ce n'est plus de la répartition des revenus qu'il s'agit, mais de la répartition des résidus au sein des élites gouvernementales et non-gouvernementales, qui décide de la dynamique sociale, politique et économique des nations.

\section{SOCIOLOGIE ÉCONOMIQUE ET LA COURBE DES REVENUS}

La courbe des revenus n'est pas prise en considération dans les ouvrages qui se sont intéressés à l'histoire de la sociologie économique (Swedberg, 1987; Gislain et Steiner, 1995).

Cette absence tient d’abord au fait que Pareto propose une démarche qui n’a pas fait école en sociologie économique, contrairement à Émile Durkheim et Max Weber, ses deux grands contemporains. ${ }^{2}$ Cela tient ensuite au fait que Pareto a choisi de mettre l'accent sur le protectionnisme douanier, un phénomène connexe

2 Pour aller à l'essentiel, on peut caractériser ces trois approches de la sociologie économique de la manière suivante (Steiner, 1999, chap. I): Durkheim adopte une posture critique et propose de remplacer l'économie politique par une sociologie économique qui prendrait 
qui prend une place importante dans l'ensemble de ses écrits et lorsqu'il sagit de développer sa sociologie économique dans le Traité. ${ }^{3}$ La manière dont Pareto étudie le protectionnisme montre ce qu'est sa démarche en sociologie économique.

Pareto emploie une méthode par « approximations successives ». Le premier pas de l'explication est celui de l'économie politique pure, à l'occasion de laquelle il suppose des individus agissant d'une manière logique et dont l'économie est le seul horizon. Dans ce cadre, l'usage des mathématiques permet d'obtenir des connaissances précises mais abstraites; aussi ne peuvent-elles suffire à rendre compte des phénomènes économiques concrets. Pour aller plus loin, Pareto passe à l'économie politique appliquée, dans laquelle il suppose toujours des individus uniquement préoccupés par les questions économiques, mais qui agissent d'une manière plus complexe car leurs passions entrent désormais en ligne de compte: à côté d’actions logiques prennent donc place une foule nombreuse d’actions non-logiques. La dernière étape de cette méthode vient avec la sociologie - ici la sociologie économique - dans laquelle il impute aux individus une pluralité de préoccupations et d'intérêts en plus de ceux associés à la richesse ; les passions et les actions non-logiques se multiplient en proportion.

Pareto a exposé cette méthode de nombreuses fois et il a fréquemment exhorté ses collègues économistes à la mettre en œuvre pour éviter de croire que ce qu'ils démontraient en tant qu'économistes purs et/ou appliqués suffisait à comprendre le phénomène économique concret - pour reprendre l'expression qui donne le titre du dernier chapitre du Manuel. Comment ce schéma est-il mis en ouvre par Pareto?

comme objets les institutions économiques; Weber accepte de son côté les résultats abstraits de la théorie économique (autrichienne), mais demande qu'ils soient complétés par des recherches sur les conditions sociales de réalisation de l'agir rationnel et qu'ils soient confrontés aux données empiriques tirés de l'histoire. Pareto propose quant à lui de complexifier progressivement la réflexion en passant d'un acteur économique rationnel (logique selon son vocabulaire) à un acteur économique et passionné, puis, finalement, à un acteur social rationnel parfois, mais passionné surtout.

3 La réflexion de Pareto sur cette question qui traverse toute son œuvre a été explicitée par ailleurs (Steiner, 1995 ; 2006). 
Lorsqu'il s'agit du protectionnisme, Pareto parcourt les trois étapes de sa méthode. Ses articles théoriques sur l'équilibre dans une économie ouverte, parus dans le Giornali degli economisti au début des années i89o et dont le contenu est repris dans l'exposé de la théorie de l'équilibre général du Cours, démontrent que le libre-échange est la politique économique à même d’assurer une création maximale de richesse. Cette démonstration d'économie pure est un point acquis à ses yeux; Pareto ne la remet jamais en cause. L'étape suivante entraîne cependant une série de changements importants. L'irruption des passions économiques et des institutions propres à leur déploiement - l'État et les banques tout particulièrement -, explique, selon Pareto, la formation d'une demande et d'une offre de protection: la première émane des entrepreneurs, dont les activités sont rendues plus lucratives par le recours à des droits protecteurs que par une amélioration des conditions de la production, tandis que la seconde vient de la classe politique, laquelle cherche un moyen de financer les dépenses publiques qu’elle propose de réaliser dans un but électoraliste. Spoliés par la protection douanière que les politiques accordent à certains entrepreneurs, les consommateurs ne réagissent pas, soit par pure et simple ignorance, soit parce qu'ils sont pris au piège du paradoxe de l'action collective que Pareto explicite à cette occasion. L'économie politique appliquée se rapproche ainsi de l'explication du phénomène économique concret, c'est-à-dire de la montée du protectionnisme européen à la fin du XIX siècle. La sociologie économique intervient ensuite: l'idée majeure de Pareto tient dans la prise en compte des effets indirects du protectionnisme ou, plus précisément, des effets économiques résultant des effets sociaux qui font suite à la mise en place de la protection douanière. En effet, dit Pareto, la possibilité d'obtenir des gains grâce à la protection douanière favorise les individus habiles à profiter des circonstances, les individus dont l'activité résulte de «l'instinct de combinaison» au détriment de ceux, plus légalistes, moins manœuvriers, mus par «la persistance des agrégats». Les «renards » ou «spéculateurs» l'emportant sur les «lions» ou «rentiers», le protectionnisme s'installe et, à sa suite, la production de la richesse diminue. S'en tenir à ce seul résultat serait cependant insuffisant. Cette victoire à des conséquences profondes puisque elle modifie progressivement le rapport entre 
les deux principales classes de résidu au sein de l'élite gouvernementale : à long terme, la présence accrue de «spéculateurs» habiles au sein de l'élite peut conduire à une production de richesse supérieure à celle qu'une élite composée de «rentiers » pourrait permettre d'atteindre, y compris dans le cas d'un maintien du libre-échange. Cette effet économique indirect permet, selon Pareto, de comprendre que l'Allemagne s'enrichisse et se développe économiquement alors même que le protectionnisme s'installe dans le pays : les «spéculateurs » protectionnistes de la Ruhr valent mieux de ce point de vue que les Junkers de l’Est, agrariens et libre échangistes!

Peut-on trouver un tel cheminement dans l'œuvre de Pareto à propos de la courbe des revenus? Tel n'est pas le cas. Pour commencer, Pareto n'entreprend jamais de rendre compte des mécanismes économiques à l'origine des très hauts revenus, sans doute parce que ces derniers ne sont à ses yeux rien d’autre que l'application directe de la concurrence sur le marché du travail, lequel valorise très fortement les compétences exceptionnelles. La partie non salariale de ces revenus s'explique, quant à elle, par l'accumulation des capitaux et l'habileté mise en œuvre dans les placements de ces derniers. Ensuite, Pareto ne fait jamais figurer dans son économie politique appliquée la question de savoir comment l'État, le système bancaire, la fiscalité, et toutes les institutions perturbatrices des résultats démontrés par l'économie pure, sont susceptibles de modifier la courbe des revenus tenue pour un invariant social alors même que ses travaux statistiques font apparaître des différences dans les pentes des courbes. En lieu et place, Pareto fait un usage polémique de sa découverte contre ceux qui cherchent à limiter les inégalités ou, pire, qui prétendent marcher vers l'égalité économique. La forme de la courbe des revenus est, selon lui, une caractéristique stable des sociétés, de toutes les sociétés; tout changement qui lui est apporté ne produit qu'une déformation passagère avant que la fine pointe des très riches et la vaste masse des pauvres reprennent leurs places dictées par les inégalités de tous genres qui hiérarchisent les individus dans la société. C'est là le résultat central auquel Pareto se tient. Enfin, il ne met pas la question de la répartition des revenus au cœur de sa sociologie et de la circulation des élites. Les modifications dans les proportions entre les différentes classes de 
résidu au sein des élites gouvernementales et non-gouvernementales résultent d'une multitude de phénomènes, dont ceux liés à la répartition, mais sans que ces derniers ne soient étudiés en tant que tels. Dans le Traité c'est la question du protectionnisme qui est, une nouvelle fois, longuement développée à ce propos.

Cela suffit-il à exclure la courbe des revenus de la sociologie économique parétienne, voire de la sociologie économique tout court? Non : les problèmes posés par la répartition des revenus, notamment en sa pointe, en liaison avec les modes de sélection des élites économiques, sont un sujet majeur de la science sociale, hier comme aujourd'hui. Si Pareto n'a pas poursuivi dans ce sens, son œuvre fournit des ressources utiles pour comprendre les changements opérés depuis trois décennies dans les sociétés contemporaines.

\section{LA «POINTE»DE LA COURBE DES REVENUS}

Deux changements majeurs se sont fait jour à ce propos. Tout d'abord, une théorie pure a été élaborée pour rendre compte des très hauts revenus. Les deux contributions majeures ont été faites par Sherwin Rosen (198I) pour l'économie des superstars et par Edward Lazear et Sherwin Rosen (198I) pour la théorie des tournois. Dans le premier cas, il s'agit d'expliquer comment des différences très faibles entre des individus détenteurs de talents particulièrement rares et demandés donnent lieu à des différences de rémunération disproportionnées; l'argument repose alors sur les modifications introduites par les technologies de communication qui permettent une diffusion mondiale des produits au profit quasi exclusif de l'individu le plus talentueux qui «ramasse alors toute la mise». Dans le second cas, les très hauts revenus sont expliqués par le fait que, survivant d'une série d'épreuves, le vainqueur reçoit une rémunération rétribuant ses efforts réalisés pour parvenir à cette fin tout au long de sa carrière et indépendamment du surcroît de productivité qu'il peut apporter à l'organisation. La théorie pure des hauts revenus que Pareto n’avait pas cherché à établir est ainsi en place. D’un strict point de vue parétien qui exige de ne pas surestimer les effets des idéologies ou encore des «dérivations», ce n’est pas l'essentiel. En effet, à côté de ces travaux, il existe toute une littérature qui s'est 
emparée de la courbe des revenus pour examiner ce qu'il en est de la montée des très hauts revenus et, par voie de conséquences, des inégalités salariales.

Les travaux que conduit Thomas Piketty $(1998$; 2003) et ses collègues (Piketty et Saez, 2003; Atkinson, Piketty et Saez, 2006; Facundo; Atkinson, Piketty et Saez, 20I3) prennent leur racines dans les travaux de Pareto.

D’une part, il s’agit de recherches économiques solidement adossées sur des séries de données décrivant finement les revenus et leurs évolutions sur la longue durée; et ces données sont examinées à la lumière de la courbe parétienne des revenus. Piketty prend d’ailleurs la peine de commenter certains des travaux de Pareto sur le sujet - en fait essentiellement l'exposition donnée dans le Cours - pour en souligner les incertitudes quant à la question de la diminution des inégalités et quant aux effets possibles d'une redistribution par l'intermédiaire de l'impôt progressif (Piketty, 1998, p. 528-529). Dans une annexe de l'ouvrage, il discute longuement la manière d'estimer les coefficients de la courbe de Pareto pour les hauts revenus, c'est-à-dire les revenus des $10 \%$ les mieux payés de la population (ibid., p. 592 et suiv.). Sa conclusion montre qu'un siècle après sa mise au jour la courbe de l'économiste de Lausanne reste tout à fait pertinente :

[... l l'hypothèse de Pareto est globalement bien vérifiée par les données fiscales françaises, et ce pour l'ensemble des revenus des années 1915-1998: les hauts revenus déclarés à l'impôt progressif sur le revenu sont caractérisés par des coefficients de Pareto de l'ordre de $b_{i}=2, \mathrm{I}-2,2$ dans l'entre-deux guerres (avec des coefficients supérieurs à 2,3-2,4 à la fin des années 1910 et au début des années 1920, puis une forte tendance à la baisse durant les années 1920-1930, et surtout durant les années de la Seconde Guerre mondiale, avec un minimum absolu inférieur à I,6 en 1944), puis de l'ordre de $b_{\mathrm{i}}=$ I,7-1,8 depuis la Seconde Guerre mondiale (ibid., p. 594).

En outre, comme il dispose d'informations bien plus précises que celles que Pareto pouvait réunir, Piketty indique que la fiabilité de l'approximation des revenus par l'estimation des coefficients de la courbe de Pareto est très bonne avec une erreur de l'ordre de $0,5 \%$ et une erreur maximale de l'ordre de I à $2 \%$ pour la partie la plus fine de la pointe de la distribution - les ०,0I \% $\left(\mathrm{P}_{99,99}\right)$ - (ibid. p. 600). 
D’autre part, ces études renouvellent les questions posées par l'inégalité dans la mesure où elles délaissent l'inégalité définie en termes de valeurs centrales (salaire moyen ou salaire médian), ou de rapport entre les extrêmes de la distribution $\left(\mathrm{P}_{90} / \mathrm{P}_{10}\right.$, soit le rapport du dixième de la population le mieux rémunéré au dixième qui l'est le moins bien) de façon à focaliser le regard sur ce qu'il se passe à l'intérieur de la «pointe » de la distribution : les I० \% les mieux rémunérés, mais surtout le I \%, le 0,I \%, voire le ०,OI \% situés à l'extrême pointe de la courbe. Les résultats sont saisissants. Piketty montre que le premier centile voit son emprise sur les flux annuels de richesses se réduire considérablement après-guerre en passant de $20 \%$ avant la Seconde Guerre mondiale à 7-8\% dans les années 1990. Il explique cette évolution par la conjonction de deux phénomènes: la chute des revenus des capitaux suite aux destructions occasionnées par la guerre, et la mise en place d'une fiscalité progressive caractérisée par des taux marginaux d’imposition élevés. Depuis lors, les très hauts revenus ont repris une partie du terrain perdu, car leur taux de croissance est beaucoup plus rapide que celui des revenus ordinaires (les premiers 90\% de la population) : sur la période 1998-2005, ces derniers n’ont crû que de 4,6\%, alors qu'ils ont progressé de $19,4 \%$ pour le premier centile, de $32 \%$ pour le premier millime et de 42,6\% pour le premier dix-millime (Landais, 2007).

Pour rendre compte de cette évolution dans le cas de la France, Piketty avance l'idée selon laquelle « [...] un très large consensus a toujours entouré ces hiérarchies salariales: l’inégalité des salaires n’a jamais été véritablement remise en cause par quelque mouvement politique que ce soit» (1998, p. 547). Ce sont les revenus des capitaux qui ont focalisé l'attention lors des luttes politiques et non les revenus salariaux, considérés comme mérités et cela d’autant plus qu'on constatait une diminution du ratio $\mathrm{P}_{90} / \mathrm{P}_{10}$ et une remarquable stabilité des seuils $\mathrm{P}_{10}$ et $\mathrm{P}_{90}$ du milieu des années 1960 à la fin du siècle, ce qui fait que le salarié appartenant au haut de la hiérarchie gagne environ 5 fois ce que perçoit celui situé en bas (ibid., p. 212-2I3). 
L'hypothèse d'un consensus sur cette distribution fortement inégalitaire des revenus peut-elle être maintenue au début du xxi siècle? Pour les revenus ordinaires - ceux qui ne dépassent pas 8000 euros mensuels, soit environ $97 \%$ de la population française - l'argument reste valable. Mais il n'en va pas de même pour les revenus perçus par le premier centile, lorsque l'on examine les réactions qui se sont faites jour à la suite de la crise financière de 2008. En effet, en suivant systématiquement la manière dont deux journaux français (Sud-Ouest pour la province, Le Monde pour la capitale) ont rendu compte du déroulement de la crise financière entre 2008 et 2010 on voit combien le contraste entre les rémunérations perçues par les dirigeants des grandes entreprises, des banques et des financiers et l'échec de ces mêmes dirigeants à faire fonctionner et à réguler le système bancaire, financier et boursier est perçu comme injuste (Steiner, 20ı I, chap.3). Disproportion, déconnexion, inégalités : tels sont les termes qui résument le point de vue diffusé par ces média et qui sidèrent jusquaux dirigeants des grandes entreprises de la génération passée, tel Georges Pébereau, ancien P-DG d’Alcatel qui s'inquiète dans les colonnes du Monde (I7 septembre 2008) de la situation, prérévolutionnaire selon ses termes, engendrée par de telles inégalités. Doit-on, en suivant le sentiment de Pareto quant à la faiblesse de l'impact des «dérivations » sur l'évolution sociale, négliger ce type de réaction? Ce n’est pas si simple, car Pareto a toujours fait valoir l'idée selon laquelle la spoliation demande que soient avancées des «prétextes» à défaut de quoi les individus dont le sentiment de justice est froissé peuvent se mettre à agir au nom de leurs principes, fut-ce au prix de leur intérêt économique. ${ }^{4} \mathrm{Si}$ tel était le cas, les effets sociaux découlant de ce qui se passe à la pointe de la courbe des revenus auraient des effets sur la forme de cette dernière, en relevant les taux marginaux d'imposition et en mettant en place des contraintes en matière de rémunération des dirigeants (vote des actionnaires, interdiction des «parachutes dorés») et des traders (limitation des bonus). Une dynamique typique de la sociologie économique telle que Pareto l'envisageait.

4 On oublie trop souvent que Pareto tient compte des réactions morales des individus au point que ces derniers peuvent agir contre leur intérêt économique: «ll faut naturellement un prétexte pour cette appropriation [spoliation par l'intermédiaire du protectionnisme], car sinon un certain instinct d'équité et de justice, qui existe dans tous les hommes, les porteraient à résister, même indépendamment de la perte dont ils sont menacés» (1964 [1896-1897], p. 382-383, §1046). 


\section{CIRCULATION DES ÉLITES?}

Les travaux de Piketty sont essentiellement tournés vers la mesure de l'inégalité des revenus, y compris ceux en provenance du capital. Ce faisant, on pourrait penser que la dynamique sociale qui intéressait tant Pareto lorsqu'il interprétait sa courbe est négligée dans le cas particulier de la circulation des élites. Tel n'est pas le cas. Dans ses premiers travaux Piketty a accordé une grande attention à l'origine des revenus qui propulsent les individus dans le petit monde des «très hauts revenus » : s'agit-il de revenus salariaux ou de revenus du capital ? L'étude de la composition des revenus perçus à la pointe de courbe montre que la partie salariale de ceux-ci a pris une ampleur qu'elle n'avait pas au début du $\mathrm{xx}^{\mathrm{e}}$ siècle, même si c'est dans cette pointe que se concentrent les revenus des capitaux. Cette modification que résume l'expression «Working Rich» (Godechot, 2007), suggère l'existence de nouvelles trajectoires menant à la fine pointe de la distribution des revenus.

Ainsi des travaux montrent l'existence d'un renouvellement dans la composition socio-économique de l'«élite du revenu ». Le travail de Thomas Philippon et Ariel Reshef (2009) sur les rémunérations dans le secteur financier américain est instructif à un double point de vue. Les deux auteurs montrent la complexité accrue du travail au sein du monde financier à partir des années I980 et donc l’accroissement du capital humain qui y est employé. Cette évolution est fortement corrélée à la dérégulation du secteur, laquelle permet à l'innovation financière de se déployer grâce aux talents qui y trouvent le moyen de s'exprimer. Cela montre qu'il existe une base économique classique - l'offre et la demande de capital humain - à l'origine du développement de ce secteur et des hauts revenus qui y sont distribués. Sur la base d'une comparaison avec des activités requérant un même niveau de capital humain, et en contrôlant les niveaux de risque encourus par les salariés, ils montrent également que les rémunérations dans le secteur financier sont supérieures à celles que produirait un équilibre durable du marché du travail. En d'autres termes, la supériorité des rémunérations dans le secteur financier vis-à-vis du secteur privé (hors agriculture) s'explique pour partie par la capacité du monde de la finance à prélever des rentes sur le reste de l'économie. 
Quels effets sociaux peuvent surgir de telles situations? Philippon et Reshel (ibid., fig. 7) en donnent une traduction éclairante en considérant le différentiel de revenu des personnes ayant une même qualification universitaire (Graduates et post-Graduates) selon qu'ils travaillent comme ingénieurs ou financiers. Pour les Graduates, le secteur financier est moins rémunérateur (de l'ordre de ıo à $20 \%$ ) jusqu'au milieu des années 1990 où la parité est atteinte, avant qu'il ne devienne légèrement plus rémunérateur (environ $5 \%$ ) à partir des années 2000. Pour les post-Graduates l'avantage offert par le secteur financier est plus ancien puisque la parité qui existait jusqu’au début des années 1980 s'efface avec un écart rapidement croissant pour atteindre, autour des années 2000, des différences de l'ordre de 10000 à 30000 \$ par an (soit I2 à $30 \%$ de plus pour un financier que pour un ingénieur de même qualification). On comprend que la National Academy of Science s'inquiète du déplacement massif des scientifiques vers le monde de la finance au détriment des autres secteurs de l'économie - phénomène qui n’est bien sûr pas spécifique aux États-Unis. À l'autre bout de la chaîne, le travail d'Olivier Godechot (20II) sur la composition professionnelle du petit monde des très hauts revenus en France montre les effets de ces très hauts revenus du monde de la finance et des services aux entreprises sur la composition de l'élite des revenus. En 1976, $38 \%$ des personnes situées dans le premier millime venaient de l'industrie, et seulement respectivement io et $6 \%$ travaillaient dans le service aux entreprises et dans la finance. Trente ans plus tard, le changement est massif: le monde de l'entreprise ne représente plus que $14 \%$ de ce premier millime, tandis que le monde des services et celui de la finance se taillent la part du lion avec, respectivement, 26 et $24 \%$ des places (Godechot, 20 I I, p. I I-I 2 et fig. 6). Compte tenu de la faiblesse du nombre de personnes travaillant dans la finance - environ $3 \%$ de l'emploi sur la période - Godechot montre qu'il existe une forte surreprésentation de la finance dans le monde des très hauts revenus. La montée des inégalités est donc fortement corrélée à l'ascension de la finance et à un renouvellement de l'élite des revenus. 
Il y a de bonnes raisons de penser que cette transformation de la composition sociale de l'élite des revenus engendre un changement durable. Les très hauts revenus perçus sur plusieurs années permettent d'accumuler d'importants patrimoines, et cela d'autant plus que l'on observe une baisse des taux marginaux d'imposition dans les pays développés depuis les années 1980. Comme la baisse affecte également la fiscalité successorale, la constitution de fortunes dynastiques devient une caractéristique majeure du capitalisme en ce début du Xxi ${ }^{\text {e }}$ siècle. En France, les flux de richesses transmis par donation et héritage atteignent désormais I $5 \%$ du revenu national, soit une multiplication par 3 en regard de ce qui avait lieu à la sortie de la Seconde Guerre mondiale. Les calculs et les simulations réalisés par Piketty (20 I) montrent que si le taux de croissance réel de l'économie reste significativement inférieur au taux de rendement des actifs, les montants ainsi transmis annuellement par donation et héritage retrouveront les hauts niveaux qui avaient été les leurs au début du $\mathrm{XX}^{\mathrm{e}}$ siècle, soit $20-25 \%$ du revenu national.

De tels résultats ne peuvent pas ne pas retenir l'attention du sociologue économiste. Conformément à l'intuition de Pareto, il y a bien des mouvements forts au sein de la pointe de la distribution des revenus, mouvements que l'on peut interpréter en termes de circulation des élites pour autant que l'on montre que les propriétés sociales de ces nouveaux entrants sont différentes de celles de leurs prédécesseurs. Trois phénomènes économiques majeurs - la montée de la finance et des rémunérations très élevées qu’elle verse à ses meilleurs éléments suite aux rentes prélevées sur l'économie réelle ; la baisse des taux marginaux d'imposition; la baisse de la fiscalité successorale - combinent leurs effets pour donner naissance à une nouvelle composante de l'élite économique dont «l'accumulation primitive» repose sur le versement de salaires et de bonus. Dans une perspective parétienne, on doit donc s'interroger sur les conséquences sociales et politiques de cette transformation, puis sur leurs conséquences économiques. L’emprise de la finance sur l'économie est désormais un fait économique et politique majeur, comme la récente série de crises financières (de la nouvelle économie en 200I, des subprimes en 2007-2008) le prouve. L'impact social et politique de la finance 
est lui aussi central. Les entreprises ont désormais fait une large place aux financiers dans leurs équipes dirigeantes (Fligstein, 1990; Zorn, 2004); les acteurs politiques majeurs - ministres, directeurs de banques centrales - sont, en Europe comme aux États-Unis, directement issus du milieu de la finance qu'ils sont censés réguler. On le voit également dans la diffusion d'une nouvelle forme d'affiliation politique qu’André Orléan (1999, chap. 4) a dénommé l’individualisme patrimonial. En termes de gestion de leur patrimoine, les individus sont de fait mondialisés et financiarisés, de telle manière que leur horizon de référence n'a plus de raison de s'arrêter au cadre national dans lequel ils sont nés et où ils travaillent; la liquidité que la finance donne à leurs actifs leur permet précisément d'échapper à cette fixation géographique et politique. Cette extraterritorialité économique sert ainsi de levier contre l'État-nation, notamment dès lors qu'il est question de réguler le marché financier ou d'imposer les revenus du capital quand bien même l'État agit en tant que «préteur en dernier ressort» dès lors que la finance entre en crise. Ces modifications sociales et politiques posent donc un défi à la science sociale.

Si la composition professionnelle des élites du revenu change indubitablement, il faudrait cependant des études plus détaillées sur les trajectoires sociales avant de pouvoir conclure à une modification de la composition sociale des élites au sens où Pareto envisageait celle-ci. En effet, si dans ses débuts, la financiarisation a pu bousculer le monde feutré de la banque d'affaire - les inventeurs des OPA financés par des «obligations pourries» n'étaient pas issus de Harvard, de Eton ou de l'École Polytechnique - on peut se demander si, quelques années plus tard, les élites sociales n’ont pas repris la main. En France comme aux États-Unis, les traders sortent des meilleures écoles, lesquelles sont les lieux par excellence de la reproduction sociale. Plus que d'une circulation des élites, on assiste actuellement à une modification des voies d'accès à l'élite des revenus et à la modification du «type d'homme» sélectionné et valorisé dans la société. Si tel est le cas, l’interrogation politique sur laquelle débouche la sociologie économique de la répartition change, mais ne perd nullement de son importance. Elle ressortit de ce que Max Weber appelait le problème de la sélection du type d'homme dominant - ou encore la question dite du Menschentum. 
En plusieurs occasions, le sociologue allemand soulignait que le problème qui l'intéressait avant tout en tant qu'économiste était celui du type d'homme que les mécanismes - ici économiques - de sélection produisaient et qui, finalement, caractérisaient un système social ${ }^{5}$ ou, plus simplement, une période et un pays ${ }^{6}$. L'examen de ce qu'il se passe à la pointe de la courbe des revenus est un moyen privilégié pour saisir un tel phénomène dans la période présente.

\section{CONCLUSION}

La courbe des revenus est un des résultats majeurs de l'économie politique appliquée de Pareto. Un siècle après, elle demeure un outil indispensable pour comprendre l'évolution des sociétés capitalistes. Les éléments que nous avons rassemblés montrent qu'elle prend également toute sa place pour comprendre les évolutions sociales et politiques que la sociologie économique prend pour objet.

L'ironie est que cet outil sert plus à montrer l'emprise de l'élite des revenus sur la société et l’injustice de la situation ainsi créée qu’à justifier des inégalités que, pour son compte, Pareto n’avait aucunement en vue de mettre en cause!

5 C'est ce qu'il explique dans une de ses anti-critiques: «Enfin, cette composante [la recherche du plus gros gain possible] de l'"esprit" capitaliste des temps modernes, que j'ai analysé en particulier - l'idée du "devoir ordonné à la profession-vocation" et tout ce qui lui est lié -, ne se trouve à son tour, au sein de l'économie portée par l'“esprit" du capitalisme, qu'à une période historique précise. Par ailleurs, cette composante s'étend, au-delà du domaine économique, à des sphères très hétérogènes de l'activité humaine. Le développement du "type d'humanité ordonnée à la profession-vocation" dans sa signification de composante de l'“esprit" capitaliste - tel est le thème auquel mes études se sont limitées pour commencer et ce expressément et volontairement» (Weber, 2003 [1910], p. 379).

6 «En économie politique, on a, de temps en temps, mis en avant ou naïvement assimilé au titre de mesure de valeur le problème technico-économique de la production des biens, et le problème de leur répartition, de la "justice sociale", et pourtant, par-dessus cette problématique qui s'est toujours et sans cesse affirmée, la certitude à demi consciente et néanmoins impérieuse qu'une science humaine - et l'économie politique en est une - s'interroge avant tout sur la qualité des hommes qui sont éduqués dans ces conditions d'existence économiques et sociales» (Weber, 1989 [1895], p. 47). 


\section{BIBLIOGRAPHIE}

BARBUT Marc, 2007, La Mesure des inégalités, Genève, Droz.

FACUNDO Alvaredo, ATKINSON John, PIKETTY Thomas, SAEZ Emmanuel, 20I3, «The Top I Percent in International and Historical Perspectives», Journal of Economic perspectives, 27-3, p. I-21.

FLIGSTEIN Neil, 1990, The Transformation of Corporate Control, Cambridge Mass., Harvard University Press.

GISLAIN Jean-Jacques, STEINER Philippe, 1995, La Sociologie économique (I8901920): Durkheim, Pareto, Schumpeter, Simiand, Veblen et Weber, Paris, PUF.

GODECHOT Oliver, 2007, Working Rich. Salaires, bonus et appropriation du profit dans l'industrie financière, La Découverte.

-, 20II, «Finance and the Rise of Inequalities in France», Paris School of Economics, Working Paper.

LANDAIS Camille, 2007, «Les hauts revenus en France (1998-2006): une explosion des inégalités?», Paris School of Economics, Working Paper.

LAZEAR Edward, ROSEN Sherwin, 1981, «Rank-Order Tournaments as Optimum Labor Contracts», Journal of Political Economy, 89-5, p. 84I-864.

ORLÉAN André, 1999, Le Pouvoir de la finance, Paris, Odile Jacob.

PARETO Vilfredo, 1964 (1896-1897), Cours d'Économie politique, Fuvres complètes, t.I-II, Genève, Droz.

-, 1965 (1901-1902), Les Systèmes socialistes, Cuures complètes, t.V, Genève, Droz.

-, 1966 (1909), Manuel d'Économie politique, CEuvres complètes, t. VII, Genève, Droz.

-, 1968 (1916), Traité de sociologie générale, Euvres complètes, t. XII, Genève, Droz.

PHILIPPON Thomas, RESHEF Ariel, 2009, «Wages and Human Capital in the U.S. Financial Industry», NBER Working Paper.

PIKETTY Thomas, 200I, Les Hauts Revenus en France au Xx siècle. Inégalités et redistribution 1901-1998, Paris, Grasset.

-, 2003, «Income inequality in France, 190 I-1998», Journal of Political Economy, III-5, p. 1004-1042. 
-, 201I, «On the Long-Run Evolution of Inheritance: France |820-2050», Quarterly Journal of Economics, 126-3, p. I07I-1/31.

-, SAEZ Emmanuel, 2003, «Income Inequality in the United States, 1913-1998», Quarterly Journal of Economics, II8-I, p. I-39.

ROSEN Sherwin, 1981, «The Economics of Superstars», American Economic Review, 7I-5, p. $845-858$.

STEINER Philippe, 1995, «Viffredo Pareto et le protectionnisme: L'économie politique appliquée, la sociologie générale et quelques paradoxes», Revue économique, 46-5, p. 1241-1262.

-, 2006, «Pareto, l'économie politique et la révision du libéralisme économique classique», in Histoire du libéralisme en Europe, Philippe Némo, Jean Petitot (éds), Paris, PUF, p. 595-616.

-, 2011, Les Rémunérations obscènes, Paris, La Découverte.

-, 2011 (1999), La Sociologie économique, 4édition, Paris, La Découverte.

SWEDBERG Richard, 1987 (1994), Pour une histoire de la sociologie économique, Paris, Desclée de Brouwer.

WEBER Max, 1989 (I895), «L'État national et la politique économique», La revue du MAUSS, 3, p. 35-59.

-, 2003 (1910), «Anticritique à propos de l'"esprit" du capitalisme» in Max Weber, L'Éthique protestante et l'esprit du capitalisme, Paris, Gallimard.

ZORN Dirk, 2004, «Here a Chief, There a Chief: the Rise of the CFO in the American Firm », American Sociological Journal, 69-3, p. 345-364. 
